\title{
Cost-Utility of Prostaglandin Analogues Compared to Beta Blockers as First-Line Medication for Treating Primary Open Angle Glaucoma in Ghana
}

\author{
Enock Takyi \\ Department of Health Management and Health Economics, University of Oslo, Oslo, Norway \\ Email: ekt1082@yahoo.co.uk
}

How to cite this paper: Takyi, E. (2017) Cost-Utility of Prostaglandin Analogues Compared to Beta Blockers as First-Line Medication for Treating Primary Open Angle Glaucoma in Ghana. Modern Economy, 8, 1231-1257.

https://doi.org/10.4236/me.2017.811084

Received: July 16, 2017

Accepted: November 3, 2017

Published: November 6, 2017

Copyright $\odot 2017$ by author and Scientific Research Publishing Inc. This work is licensed under the Creative Commons Attribution International License (CC BY 4.0).

http://creativecommons.org/licenses/by/4.0/ (c) (i) Open Access

\begin{abstract}
Ghana ranks among the countries most affected by glaucoma in the world. It is estimated that 700,000 people in Ghana are affected by glaucoma, out of which 60,000 people are already blind. In recent years, there has been a growing concern that anti-glaucoma medications listed on the country's health insurance medicines list are not adequate to manage the disease. This has led to calls from different stakeholders for prostaglandin analogues to be added to the medicines list. However, the cost-effectiveness of this medication is yet to be established. The objective of this study, therefore, was to establish the costeffectiveness of prostaglandin analogues as first-line medication for treating primary open angle glaucoma in Ghana. A Markov Model was constructed to assess the life-time cost-effectiveness of treating a hypothetical cohort of one thousand 55-year-old POAG patients with prostaglandin analogues compared to beta blockers. The effect of parameter uncertainty on cost-effectiveness was explored through one-way, two-way, and probabilistic sensitivity analyses. Compared to beta blockers, prostaglandin analogues resulted in an incremental cost-effectiveness ratio (ICER) of USD 11,600; based on an estimated incremental gain of 105 quality-adjusted life years (QALYs), at an additional cost of USD 1,222,400. The ICER was not sensitive to the age of the cohort, the cost of prostaglandin analogues, or the probability of developing asthma. However, the ICER was sensitive to the age of the cohort and the cost of prostaglandin analogues when varied simultaneously. Given the existing evidence, prostaglandin analogue is not a cost-effective alternative to beta blockers as a first-line treatment for POAG in Ghana. The study, however, shows that further research to reduce decision uncertainty would be necessary if expected cost of research does not exceed USD 131 billion.
\end{abstract}




\section{Keywords}

Glaucoma, Ghana, Prostaglandin Analogue, Beta Blocker, ICER, Cost-Effectiveness

\section{Introduction}

Ghana ranks among the countries most affected by glaucoma in the world [1] [2]. A recent estimate of the number of people affected by glaucoma worldwide suggested that, by 2020 there will be approximately 80 million people living with the disease, of which 11 million will be bilaterally blind [3]. In Ghana, glaucoma is estimated to account for approximately $21 \%$ of all cases of blindness [4]. It is estimated that 700,000 people in Ghana are affected by glaucoma, out of which 60,000 people are already blind [5]. Studies on incidence of glaucoma in Ghana, and Africa in general, are hard to find. Cook [6] estimated the annual incidence of glaucoma in Africa at $0.04 \%$ (i.e. 400 new cases for every 1 million population). Thus, considering Ghana's population of 27.41 million, the annual incidence of glaucoma can be estimated to be 10,964 per year. Glaucoma, with the resulting blindness, is a burden not only to patients, but to the entire society. To patients, the burden takes the form of pain and loss of function. It is also a burden to society in terms of resources used for treatment of patients, and productivity loss due to permanent disability associated with the disease.

"Glaucoma is a group of disorders, principally optic neuropathy resulting from cupping and atrophy of the optic nerve head leading to visual loss and blindness" [7]. Glaucoma is often associated with increased intraocular pressure (IOP) in the eye [8]. The higher the IOP, the greater the risk of visual loss [8]. IOP is measured in millimetres of mercury $(\mathrm{mmHg})$. In anti-glaucoma therapy, an IOP between $19 \mathrm{mmHg}$ and $22 \mathrm{mmHg}$ is considered to be the safest for patients, although there is no single IOP level that is safe for every patient [7] [9]. Diagnostically, visual field loss is the most specific sign of glaucoma [7] [10]. The visual field is the portion of an individual's surroundings that can be seen at any given point in time [11]. Visual field is measured in decibels (dB) [12] [13]. Lower $d B$ values represent lower retinal sensitivity, while higher $d B$ values represent higher retinal sensitivity [13]. There are various types of glaucoma, but the commonest in Ghana is Primary Open Angle Glaucoma (POAG) [8]. Blindness from glaucoma is irreversible; treatment for glaucoma is, therefore, meant to reduce the risk of progression [14]. IOP is an important modifiable risk factor for glaucoma [15]. It has been proven that lowering IOP reduces progression of visual field loss in glaucoma [14] [15].

The main interventions for treating glaucoma are medications, laser therapy, and surgery [15]. The aim of glaucoma treatment, often, is to achieve a pressure reduction of at least $30 \%$ from the initial IOP [9]. There are five main classes of anti-glaucoma medications: prostaglandin analogues, beta blockers, carbonic 
anhydrase inhibitors, sympathomimetics and miotics [16]. Ghana adopted a social health insurance system in 2003. The National Health Insurance Scheme (NHIS) was established by the National Health Insurance Act, 2003 (Act 650) [17]. The act also established the National Health Insurance Authority (NHIA), which regulates all health insurance schemes in the country [18]. As part of its mandate to regulate the health insurance schemes in Ghana, the NHIA has listed several anti-glaucoma medications to the health care needs of its registered clients. The NHIS medicines list includes medications extracted from all the classes of anti-glaucoma medications except prostaglandin analogues, although prostaglandin analogues are proven to be more effective [7]. This is probably because, generally, prostaglandin analogues cost more than medications from the other classes.

In recent years, there has been a growing concern that the anti-glaucoma medications listed on the country's National Health Insurance medicines list are not adequate to manage the disease. Many stakeholders have suggested that the NHIA should review the anti-glaucoma medications on the NHIS medicines list to include more medications. In their study to establish the "efficacy of NHISlisted anti-glaucoma medications in the management of primary open-angle glaucoma in Ghana", Koffour et al. [7], observed that the NHIS-listed antiglaucoma medications were not adequate in the management of POAG. The authors found that prostaglandin analogues, which were not listed by the NHIA, was more efficacious than the NHIS-listed medications. They, therefore, suggested that prostaglandin analogues, particularly latanoprost, be added to the NHIS list. A similar suggestion was made by the authors of the Ghana Eye Health System Assessment Report 2013 [1]. However, the cost-effectiveness of this medication has not yet been established. The aim of this study, therefore, was to establish the cost-effectiveness of prostaglandin analogue as a first-line medication for the management of POAG in Ghana. Currently, the main firstline anti-glaucoma medications used in Ghana are beta blockers and prostaglandin analogues (expert opinion). Thus, the study compares prostaglandin analogues to beta blockers.

Studies on glaucoma in Ghana are very scanty. Koffour et al. [7] established the efficacy of anti-glaucoma medications in Ghana, but did not account for their costs. Wittenborn and Rein [19] also compared the Cost-effectiveness of glaucoma interventions in Barbados and Ghana. However, the authors did not establish the cost-effectiveness between different ant-glaucoma medications. Thus, studies that establish the cost-effectiveness of anti-glaucoma medications in Ghana are almost non-existent. It is this gap that the current study seeks to fill.

The primary question that this study seeks to answer is: Is prostaglandin analogue a cost-effective first-line treatment for primary open angle glaucoma in Ghana?

Ghana is a country in West Africa. The World Bank estimates Ghana's population at 27.41 million [20]. According to the World Health Organization 
(WHO), the life expectancy of a Ghanaian at birth is 63 years (i.e. 64 years and 62 years for females and males respectively) [21]. The World Bank classifies Ghana as a lower middle-income country [22]. The World Bank figures from 2015 estimated Ghana's gross domestic product (GDP) at USD 37.86 billion [20]. This translates into a GDP per capita of USD 1381.40. It is estimated that 24.2 percent of Ghana's population live below the poverty line, while an estimated 8.4 percent is said to be extremely poor [23]. The most recent figures available (from 2014) indicate that Ghana spends 3.6\% of its GDP (approximately USD 1.36 billion) on health care [24].

Health care needs are insatiable, but the resources to meet these needs are always limited. It is, therefore, imperative to prioritize health care needs to get the most out of the limited resources. Findings from this study will, therefore, help Ghana's National Health Insurance Authority to make informed decision as to whether prostaglandin analogous can be adopted as a cost-effective first-line treatment for POAG in Ghana or not. Besides, the study will provide a relevant framework for future studies on glaucoma treatment costs and health gains, given that such studies are lacking in Ghana.

\section{Methods and Data}

\subsection{Markov Model}

A Markov model was developed to ascertain the cost-effectiveness of treating a hypothetical cohort of one thousand 55-year-old glaucoma patients until 80 years or death. The cohort entered the model at 55 years, because the mean age of POAG patients in Ghana was estimated to be 55 years (expert opinion). A Markov model is a mathematical method for quantifying the costs and health consequences of a disease as a patient progress through various disease stages over time. It is therefore useful when dealing with a chronic condition like glaucoma. "Glaucoma is a progressive disease where a patient's sight can deteriorate and never recover" [16]. The model is, therefore, a Markov model where patients cannot go back to previous health states. Data on intervention effectiveness and transition probabilities were taken from literature. Cost data were obtained mainly from Ghana's National Health Insurance Scheme (NHIS) medicines list for 2016 and the recently revised Ghana Diagnostic-related group (G-DRG) tariff lists. Costs of treatments not covered by the NHIS were estimated as the average of retail prices quoted by some leading pharmaceutical companies in Ghana.

The Hodapp, Parrish and Anderson classification, as adapted by the National Collaborating Centre for Acute Care (UK) [16] was used to define the POAG stages. The Hodapp, Parrish and Anderson classification classifies glaucoma based on severity of visual field defect. This staging system was adopted because it allows for utility values associated with different severity levels of POAG already present in literature [16]. Compared to the original staging system, the National Collaborating Centre for Acute Care (UK) collapsed the last two stages 
(severe POAG and blindness), on the grounds that there was an overlap of their definitions and a lack of data of progression in the absence of treatment from severe POAG to blindness [16]. The staging classification of POAG used in the study is presented in Table 1.

\subsection{The Model Structure}

Five health states were used in the model, namely, early POAG, moderate POAG, advanced POAG, severe visual impairment, and death. I assumed that the cohort entered the model at early POAG stage. During each cycle, a patient may either remain in the early POAG stage, progress to moderate POAG stage, or die. Similarly, a patient at moderate POAG stage may remain in the stage, progress to advanced POAG stage or die. A patient who begins a cycle at the advanced POAG stage may also progress to severe visual impairment, remain in the advanced POAG stage, or die. Finally, a patient with severe visual impairment may remain in that stage or die during a cycle. The structure of the Markov model is presented in Figure 1.

The cycle length was set at 2 months as this was thought to be the minimum time after which a change in treatment could occur (expert opinion). All the probabilities, costs and health utilities were, therefore, converted to reflect the two-month values. The conversion of yearly probabilities to two-month probabilities involved two steps. First, the annual probability was converted to two-month rate using Equation (1).

$$
r=-[\ln (1-p)] / t
$$

where $r=$ rate, $p=$ probability, $t=$ period of interest

The two-month rate was then converted to two-month probability by rearranging Equation (1).

$$
p=1-\exp ^{(-r t)}
$$

\subsection{Baseline Probability of Progression}

A search was conducted for studies reporting the probability for progression from one POAG stage to the next. Specifically, the search was to identify studies that reported the probability of progression from Early to Moderate POAG in

Table 1. Staging Classification for POAG.

\begin{tabular}{cc}
\hline POAG Stage & Defect Score Interval (in decibels) \\
\hline No POAG & No visual field defect \\
Early & -0.01 to $-6.00 \mathrm{~dB}$ \\
Moderate & -6.01 to $-12.00 \mathrm{~dB}$ \\
Advanced & -12.01 to $-20.00 \mathrm{~dB}$ \\
Severe Visual Impairment & $-20.01 \mathrm{~dB}$ or worse \\
\hline
\end{tabular}

Source: (National Collaborating Centre for Acute Care (UK), 2009). 


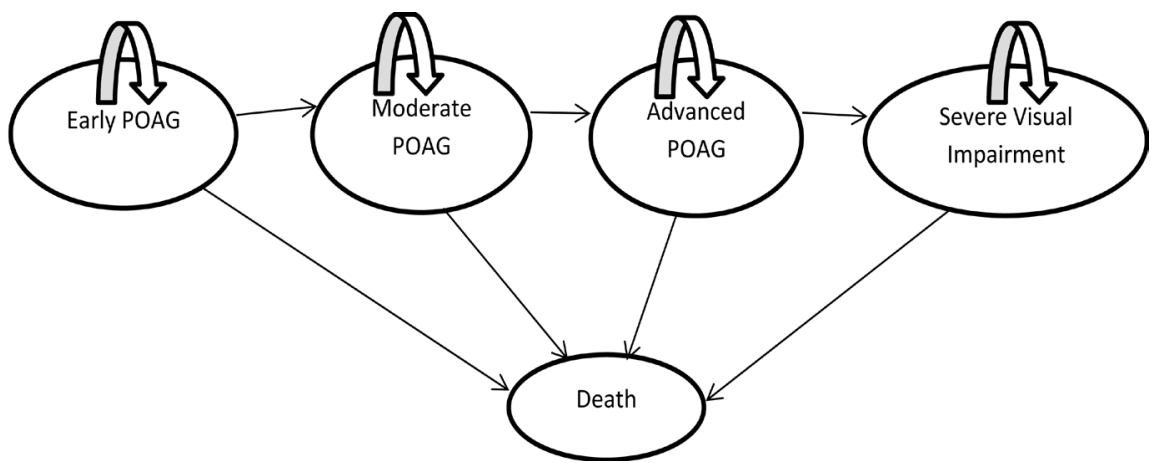

Figure 1. Structure of the markov model.

treated and untreated patients, from Moderate to Advanced POAG in treated and untreated patients, and from Advanced POAG to Severe Visual Impairment in treated and untreated patients. Only studies using a definite staging system were included. The search yielded two relevant results. Using randomized controlled trials (RCTs), Burr et al. [25] estimated the progression rates of mild, moderate, and severe POAG stages based on visual field mean defect. These stages correspond to the National Collaborating Centre for Acute Care (UK) [16] definitions of early, moderate and advanced POAG, being employed in this study. The National Collaborating Centre for Acute Care (UK) adopted Burr's progression rates, and projected the progression rate for severe visual impairment, which was missing in Burr's study. The annual progression probabilities, as presented by the National Collaborating Centre for Acute Care (UK) [16], were employed in this study, because they cover all the relevant POAG stages. The annual probabilities were converted to two-month probabilities using Equations (1) and Equation (2) as shown in Table 2.

\subsection{Treatment Effects}

Patients diagnosed with POAG could be treated either with beta-blockers or a prostaglandin analogues as first-line treatment. The main treatment effect considered was a change in risk of progression to the next POAG stages. However, the most commonly reported treatment outcome in literature is the change in intraocular pressure (IOP). Data on reduction in IOP from baseline due to each treatment was derived from the National Collaborating Centre for Acute Care (UK) [16] (Appendix 1). This was done because the data presented by the National Collaborating Centre for Acute Care was based on rigorous search for systematic reviews of clinical effectiveness of treatments in POAG patients.

Conversion of a change in IOP to a change in probability of progression involved two steps. First the change in IOP (i.e. mean reduction in IOP) associated with each intervention was multiplied by the percentage reduction in progression per $1 \mathrm{mmHg}$ reduction in IOP to obtain the effectiveness of the intervention using Equation (3). Percentage reduction in progression per $1 \mathrm{mmHg}$ reduction in IOP was taken from Leske et al. [26], who found that a $1 \mathrm{mmHg}$ reduction in IOP is associated with $8 \%$ decrease in progression from one POAG 
Table 2. Baseline probability of progression in POAG patients.

\begin{tabular}{ccccc}
\hline Transition & $\begin{array}{c}\text { Annual } \\
\text { Probability in } \\
\text { Untreated } \\
\text { Patients }\end{array}$ & $\begin{array}{c}\text { Two-Months } \\
\text { Probability } \\
\text { in Untreated } \\
\text { Patients }\end{array}$ & $\begin{array}{c}\text { Annual } \\
\text { Probability in } \\
\text { Treated Patients }\end{array}$ & $\begin{array}{c}\text { Two-Months } \\
\text { Probability in } \\
\text { Treated Patients }\end{array}$ \\
\hline Early to Moderate POAG & 0.25 & 0.048 & 0.20 & 0.037 \\
Moderate to Advanced POAG & 0.11 & 0.019 & 0.07 & 0.012 \\
$\begin{array}{c}\text { Advanced POAG to Severe } \\
\text { Visual Impairment }\end{array}$ & 0.10 & 0.018 & 0.06 & 0.01 \\
\hline
\end{tabular}

Source: (National Collaborating Centre for Acute Care (UK), 2009)

stage to the next.

$$
E f f_{i}=\Delta I O P_{i} * \Delta P
$$

where:

$$
\text { Eff } f_{i}=\text { Effectiveness of intervention } i
$$

$\triangle I O P_{i}=$ change in IOP associated with intervention $i$

$\Delta P=$ percentage change in progression associated with $1 \mathrm{mmHg}$ change in $I O P$

$i=$ beta blockers, prostaglandin analogues

Subsequently, the baseline probability of progression without treatment was adjusted by the effectiveness of the respective treatment to obtain the probability of progression after treatment using Equation (4).

$$
T P_{i}=B P u *\left(1-E f f_{i}\right)
$$

where:

$$
\begin{aligned}
& T P_{i}=\text { Transition probability associated with intervention } i \\
& B P u=\text { Baseline transition probability in untreated patients }
\end{aligned}
$$

A ratio between the probability of progression associated with prostaglandin analogues and that of beta blockers gave a relative risk of 0.843 . The probabilities are shown in Table 3.

The probabilities in Table 3 were used only in early POAG. Once a patient progressed from early POAG, the baseline probability in treated patients for the respective stage was used (Table 2). The transition probabilities for nonspecific treatments were used, because after progression any new treatment could be introduced.

Some treatments for glaucoma could cause adverse events. "Nevertheless, not all of them result in important increased costs or reduced quality of life" [16]. Asthma (associated with beta blockers) was the only complication considered in the model, because, as far as medical treatment is concerned, asthma is the only known complication with a considerable impact on costs and quality of life [16]. Probability of developing asthma after use of beta blockers was taken from a prospective cohort study [27]. The authors compared the difference in respiratory disease in 2645 patients treated with beta blockers to a control group of 9094 patients who were not exposed to beta blockers. After 12 months, it was 
Table 3. Probability of Progression after Treatment.

\begin{tabular}{cccccc}
\hline Intervention & $\begin{array}{c}\text { Annual } \\
\text { Probability } \\
\text { in Untreated } \\
\text { Patients }\end{array}$ & $\begin{array}{c}\text { Overall } \\
\text { Annual } \\
\text { Progression } \\
\text { Reduction }\end{array}$ & $\begin{array}{c}\text { Annual } \\
\text { Probability } \\
\text { of } \\
\text { Progression }\end{array}$ & $\begin{array}{c}\text { Two-Months } \\
\text { Probability } \\
\text { of } \\
\text { Progression }\end{array}$ & Relative Risk \\
\hline $\begin{array}{c}\text { Beta Blockers } \\
\text { Prostaglandin } \\
\text { Analogues }\end{array}$ & 0.25 & 0.23 & 0.193 & 0.0351 & - \\
\hline
\end{tabular}

a $0.0296 / 0.0351$.

observed that patients who were treated with beta blockers were 3.3\% more likely to develop reversible airways obstruction than those who were not exposed. This translates into a two-month probability of $0.6 \%$. The study, however, showed that the risk of respiratory problems was no longer significant after the first year of exposure. Hence the probability of developing asthma was used in the model only within the first year.

Probabilities of death were obtained from 2013 life table for Ghana as shown by WHO [28] (see Appendix 2). Life expectancy in patients with POAG was assumed to be the same as the general population in Ghana. Similarly, life expectancy in POAG patients who develop asthma due to the use of beta blockers was assumed to be the same as that of the general population in Ghana. The key parameters used in the model are summarized in Table 4.

\subsection{Treatment Outcomes}

Health gain was measured in terms of quality-adjusted life-years (QALYs). Health utilities for the health states used in the model were obtained from literature. Except for asthma, all adverse events were assumed to be negligible in terms of quality of life since, according to the National Collaborating Centre for Acute Care (UK) [16], they could be promptly treated. Disutility due to asthma was estimated from quality of life measures in treated asthma patients as presented in literature.

A search was conducted for studies reporting health utilities for patients at the various stages of POAG. The search yielded three results [16] [29] [30]. van Gestel et al. [29] could not be used because the authors did not employ the staging classification being used in this study. Similarly, Béchetoille et al. [30] could not be used, because the authors did not report the overall health related quality of life (HRQoL) for the stages; rather HRQoL was reported per different health domains. The HRQoL reported by National Collaborating Centre for Acute Care (UK) [16], which followed the same staging classification as adopted in this study, were therefore used. The National Collaborating Centre for Acute Care (UK) estimated the health utilities from Rein, Wirth, Johnson, and Lee [31], who applied utilities for visual acuity to each category of visual field loss using data obtained from Brown, Brown, Sharma, and Landy [32]. The original data was based on EQ-5D. 
Table 4. Key Parameters used in the Markov Model.

\begin{tabular}{|c|c|c|}
\hline Parameter & Value & Distribution \\
\hline Transition probability from early POAG to moderate POAG (BB) & 0.0351 & Dirichlet \\
\hline Probability of staying in early POAG (BB) & 0.9585 & Dirichlet \\
\hline Transition probability from early POAG to death (BB) & 0.0064 & Dirichlet \\
\hline Transition probability from early POAG to moderate POAG (PA) & 0.0296 & Dirichlet \\
\hline Probability of staying in early POAG (PA) & 0.964 & Dirichlet \\
\hline Transition probability from early POAG to death (PA) & 0.0064 & Dirichlet \\
\hline Transition probability from moderate POAG to advanced POAG & 0.012 & Dirichlet \\
\hline Probability of staying in moderate POAG & 0.9816 & Dirichlet \\
\hline Transition probability from moderate POAG to death & 0.0064 & Dirichlet \\
\hline $\begin{array}{l}\text { Transition probability from advanced POAG to severe visual im- } \\
\text { pairment }\end{array}$ & 0.0103 & Dirichlet \\
\hline Probability of staying in advanced POAG & 0.9834 & Dirichlet \\
\hline Transition probability from advanced POAG to death & 0.0064 & Dirichlet \\
\hline Probability of staying in severe visual impairment & 0.9937 & Beta \\
\hline Transition probability from severe visual impairment to death & 0.0064 & Beta \\
\hline Probability of asthma (BB) & 0.006 & Beta \\
\hline Probability of no asthma (BB) & 0.994 & Beta \\
\hline Two-month discount rate & 0.0025 & - \\
\hline Relative risk for prostaglandin analogues & 0.8433 & Lognormal \\
\hline Probability of needing medication in severe visual impairment & 0.1 & Beta \\
\hline Probability of not needing medication in severe visual impairment & 0.9 & Beta \\
\hline Cost of treatment for early POAG patients who use beta blockers & 3.77 & Gamma \\
\hline $\begin{array}{l}\text { Cost of treatment for early POAG patients who use prostaglandin } \\
\text { analogues }\end{array}$ & 51.54 & Gamma \\
\hline Cost of treating asthma patients & 28.78 & Gamma \\
\hline Cost of treating moderate POAG patients & 34.91 & Gamma \\
\hline Cost of treating advanced POAG patients & 54.67 & Gamma \\
\hline Cost of treating patients with severe visual impairment & 3.77 & Gamma \\
\hline Health-related quality of life for patients with early POAG & 0.989 & Lognormal \\
\hline Health-related quality of life for patients with moderate POAG & 0.944 & Lognormal \\
\hline Health-related quality of life for patients with advanced POAG & 0.819 & Lognormal \\
\hline $\begin{array}{l}\text { Health-related quality of life for patients with severe visual } \\
\text { impairment }\end{array}$ & 0.503 & Lognormal \\
\hline Health disutility due to asthma & 0.14 & Lognormal \\
\hline
\end{tabular}

$\mathrm{BB}=$ Beta blockers PA $=$ Prostaglandin analogues.

A search for quality of life measures in treated asthma patients retrieved two studies [33] [34], which respectively reported the health utility in treated asthma patients as 0.84 and 0.86 . Both studies used EQ-5D. However, I opted for Chen 
et al. [34], since it is more current. I therefore assumed that treated asthma symptoms reduce quality of life by 0.14 over one year. Health utilities used in the model are summarized in Table 4.

For each strategy, the expected health benefits were calculated by estimating the health effects for each POAG stage and then multiplying them by the proportion of patients who would be in that stage as determined by the strategy taken. The expected health benefit per the cohort in each cycle was calculated using Equation (5).

$$
\text { Expected Health Benefit }=\sum\left(Q_{i} \times P_{i}\right)
$$

where:

$Q_{i}=$ health utility of stage $\mathrm{i}$

$P_{i}=$ proportion of patients in the stage $\mathrm{i}$

$i=$ early POAG, moderate POAG, advanced POAG, severe visual impairment

The proportion of patients in each POAG stage depends on the magnitude of the progression reduction of the treatment and on the proportion of patients still alive per the mortality rate for the general population of Ghana.

\subsection{Costs}

The study adopted the health care provider's perspective. This was because the study was meant to inform the NHIA of Ghana, whose responsibility is limited to the costs incurred by the health care provider in providing services to NHIS card holders. All costs used in the model were estimated using a bottom-up approach. Follow-up costs were not considered in the model. Where the primary interest is incremental cost, as is the case when comparing the introduction of a new intervention against an existing intervention, costs that remain approximately the same for each alternative being considered can be ignored [35] [36] [37]. This is because such costs will not affect the choice between the given alternatives [35] [37]. All glaucoma patients in Ghana are followed up every two months, except a few patients whose IOP levels are exceptionally too high, or who develop intolerance to treatment before their next appointment date (expert opinion). Besides, follow-up procedures are the same for all glaucoma patients in Ghana, irrespective of their level of the disease. Hence, I assumed that follow-up cost is the same for both the intervention group and the comparator group. Since my primary interest is incremental cost, I decided not to include the follow-up cost.

Costs of all medicines were obtained from Ghana's NHIS medicines list for 2016 [38], except the cost of latanoprost which was not covered by the NHIS. Cost of latanoprost was taken as an average of the retail prices quoted by some leading pharmaceutical companies in Ghana. Two versions of latanoprost are used in Ghana, namely branded latanaprost (Xalatan) and generic latanoprost. The costs of these two versions were averaged. The cost of glaucoma surgery (trabeculectomy) was also obtained from the recently revised Ghana Diagnosticrelated group (G-DRG) tariff lists [39] [40]. The G-DRG offers different tariffs 
for services provided at Public Primary Care Hospitals and those provided at Christian Health Association of Ghana (CHAG) Primary Care Hospitals. The cost of glaucoma surgery was, therefore, taken as the average of the tariffs for Public Primary Care Hospitals and CHAG Primary Care Hospitals respectively. For purposes of comparison, all costs were presented in United States Dollars (USD), based on Bank of Ghana exchange rates for 30th June 2016 (USD 1.00 $\approx$ GHC 3.9240). The unit costs of resources used in the model are presented in Appendix 3.

Costs were categorized based on the health states used in the model. Hence the cost categories include early POAG costs, moderate POAG costs, advanced POAG costs, severe visual impairment costs, and costs of adverse events. Early POAG costs are costs incurred in treating a patient diagnosed with early POAG. They are costs that arise before progression from early POAG stage. As already mentioned, the model primarily compares the first-line anti-glaucoma medications used in Ghana (i.e. beta blockers and prostaglandin analogues). Hence, early POAG costs are those costs directly associated with either beta blockers or prostaglandin analogues as a mono therapy.

When mono-therapy fails to significantly lower IOP, combination therapy is sought for [7]. I assumed that all patients who progressed beyond early POAG were given combination therapies. Koffuor et al. [7] observed that, in Ghana, a POAG patient could be given up to four-medication combination therapies, depending on the severity of the disease and patients' tolerance to medication. However, the number of patients on four-medication combination therapy, as reported in their study, was almost insignificant. Therefore, I assumed that patients at moderate and advanced POAG stages are put on two-medication and three-medication combination therapies respectively. Besides, at both moderate and advanced POAG stages, a patient can undergo surgery (trabeculectomy). Although some patients undergo laser trabeculoplasty, the practice is not common in Ghana (expert opinion). This option was, therefore, ignored. It was assumed (based on expert opinion) that $10 \%$ of patients with severe visual impairment are given timolol to ease the pain in the affected eye; the remaining is not given further treatment.

All adverse events, except asthma, were assumed to be negligible in terms of costs. The cost of treating asthma was estimated based on the Standard Treatment Guidelines of Ghana [8]. I assumed that no additional follow-up cost was associated with the change in treatment due to asthma.

The key cost inputs used in the model are summarized in Table 4. For each strategy, the expected cost per the cohort in each cycle was calculated using Equation (6).

$$
\text { Expected cost }=\sum\left(C_{i} \times P_{i}\right)
$$

where:

$C_{i}=$ cost of stage $i$

$P_{i}=$ proportion of patients in the stage $i$ 
$\mathrm{i}$ = early POAG, moderate POAG, advanced POAG, severe visual impairment

\subsection{Analyses}

The cost-effectiveness analysis was conducted using Microsoft Excel 2016. All future costs and QALYs were discounted at the rate of 3 percent per annum in line with Global Burden of Disease [41], using Equation (7).

$$
P V=\frac{F V}{(1+r)^{n}}
$$

where:

$P V=$ Present value

$F V=$ Future value

$r=$ Discount rate

$n=$ Number of periods under consideration

Costs and health outcomes were corrected for continuity. This was done by dividing the costs and health outcomes of the first and the last cycles by 2 , as shown in Equation (8).

$$
A=1 / 2+1+1+1+\cdots+1+1+1+1 / 2
$$

where $A=$ total cost, total health effect.

The overall lifetime expected health benefit of each intervention is given by the sum of health benefits calculated for all the cycles. Since each cycle represented 2 months, the overall lifetime expected health benefit was divided by 6 to get the overall lifetime QALYs. The incremental QALY $(\Delta E)$ associated with prostaglandin analogues was calculated as the difference between the expected QALY gained with prostaglandin analogues and the expected QALY gained with the comparator (beta blockers).

Similarly, the overall lifetime expected cost of each intervention is given by the sum of costs calculated for all the cycles. The incremental cost $(\Delta C)$ associated with prostaglandin analogues was calculated as the difference between the expected cost with prostaglandin analogues and the expected cost with beta blockers.

\subsection{Incremental Cost-Effectiveness Ratio (ICER)}

The result of cost-utility analysis was expressed as incremental cost-effectiveness ratio (ICER), i.e. the ratio of incremental costs $(\Delta C)$ to incremental effects $(\Delta E)$ brought about by prostaglandin analogues compared to beta blockers. Thus, the numerator of the ICER is the difference in cost between patients in the intervention group and those in the comparator group, while the denominator is the difference in health outcomes (QALYs) between patients in the intervention group and those in the comparator group. The ICER is defined by Equation (9).

$$
\mathrm{ICER}=\Delta C / \Delta E
$$

where:

$\Delta C=$ incremental cost 


\section{$\Delta E=$ incremental effect}

To determine whether, or not, prostaglandin analogues was cost-effective, the resulting ICER was compared to the society's willingness-to-pay threshold $(\lambda)$. Prostaglandin analogue would be cost-effective only if the ICER was less than the willingness-to-pay threshold (i.e. $\Delta C / \Delta E<\lambda$ ).

The willingness-to-pay threshold for Ghana was set to be equal to three times the country's GDP per capita. Ghana's GDP per capita for 2015, according to the World Bank [20] was USD 1381.40. Hence, the willingness-to-pay threshold equals USD 4144.20 ( $\approx$ USD 4,100).

\subsection{Sensitivity Analyses}

Sensitivity analyses were performed to assess the robustness of the model results against the imprecision in the model parameters. Almost all parameters used in the model were estimates; there is uncertainty surrounding their true values. The purpose of sensitivity analyses was to capture this uncertainty in the model. One-way, two-way, and probabilistic sensitivity analyses were performed.

One-way sensitivity analysis was performed on probability of developing asthma, age of the cohort, and cost of latanoprost. I found that costs and QALYs in the comparator could be significantly influenced by asthma. For this reason, I conducted a one-way sensitivity analysis on the probability of developing asthma. In the base-case model, the annual probability of developing asthma in patients using beta blockers was set to be 3.3\%. However, in the sensitivity analysis the probability was varied between 0 and $6 \%$. Age largely influences longevity. The age at which the cohort entered the model would, therefore, determine how long the patients would live to benefit from the intervention. If the cohort dies out too quickly, the full effect of the intervention may not be realized. One-way sensitivity analysis was, therefore, performed on the age of the cohort to determine how varying the age of the cohort would affect the results. In the base-case analysis, the age of the cohort was set at 55 years. However, in the sensitivity analysis, the age of the cohort was varied between 30 years and 75 years. I also performed one-way sensitivity analysis on the cost of prostaglandin analogues (i.e. latanoprost). This was because the disparity between the cost of generic latanoprost and branded latanoprost (Xalatan) was very wide. In the base-case model, the cost per bottle of prostaglandin analogues was estimated at USD 25.77 (reflecting the mean cost of generic latanoprost and Xalatan). In the sensitivity analysis, however, the cost was varied from USD 13.00 (corresponding to the cost per bottle of generic latanoprost) to USD 39.00 (corresponding to the cost per bottle of Xalatan). A two-way sensitivity analysis was also performed to ascertain the combining effect of the age of the cohort and the cost of prostaglandin analogues on the results.

In the probabilistic sensitivity analysis (PSA), probability distributions were assigned to each model parameter where there was some degree of parameter variability. The main results were then re-calculated 1000 times; each time all the 
model parameters were set simultaneously, selecting from the respective parameter distribution at random. The decision uncertainty was represented by plotting a cost-effectiveness plane, a cost-effectiveness acceptability curve (CEAC), and a cost-effectiveness acceptability frontier (CEAF).

\subsection{Expected Value of Perfect Information (EVPI)}

Considering the uncertainty surrounding the results, it became imperative to establish whether it was worthy, or not, to gather more evidence. The EVPI was estimated directly from the results of the PSA. The EVPI is the difference between the expected net benefit with perfect information and that of current information. The EVPI was estimated using the following steps. First, the average overall net monetary benefits (NMBs) from the 1000 simulations for each intervention were calculated. With current information, the optimal decision is the intervention with the maximum expected net benefit. Afterwards, the maximum of the net benefits between the two interventions for each simulation were chosen, summed up and averaged to get the expected net benefit of a decision with perfect information. The EVPI was then estimated as the difference between the expected net benefit with perfect information and the expected net benefit with current information. The EVPI thus estimated was for a single individual.

To decide whether to invest in additional research or not, the size of the population that is expected to benefit from the new intervention and the relevant time horizon of the new intervention must be taken into account [42]. The incidence of glaucoma in Ghana was estimated at 10,964 per annum. It was assumed that 192,000 people currently living with glaucoma in Ghana (approximately $27.5 \%$ of the estimated prevalence of 700000) would also be eligible for the new intervention. It was further assumed that prostaglandin analogues would be useful for 10 years. Population EVPI was calculated as the product of the individual EVPI and the total number of people who are expected to benefit from the intervention (Equation (10)). Incidence associated with future years was discounted at the rate of $3 \%$.

$$
\text { Population EVPI }=\text { EVPI } \cdot \sum_{t=1,2, \cdots, T} I_{t} /(1+r)^{t}
$$

where:

$T=$ expected lifetime of the new intervention

$I_{t}=$ estimated incidence of the disease over the period

$r=$ discount rate

Population EVPI estimates were calculated over a cost-effectiveness threshold range of USD 0 to USD 60,100 per QALY. As a decision rule, further research is potentially cost-effective if population EVPI exceeds the expected cost of additional research at any given threshold.

\section{Results}

\subsection{Base-Case Cost-Effectiveness Analysis Results}

The expected cost of treating 1000 patient with prostaglandin analogues (USD 
$3,886,100$ ) is higher compared to beta blockers (USD 2,663,700). Similarly, QALY gained from prostaglandin analogues $(14,231)$ is higher compared to beta blockers $(14,126)$. The incremental cost-effectiveness ratio (ICER) is USD 11,600 per QALY gained (Table 5).

This implies that that if we choose prostaglandin analogues as first line treatment for POAG patients in Ghana instead of beta blockers, we would gain additional QALYs at an additional cost of USD 11,600 per each QALY gained. The ICER is greater than three times the GDP per capita of Ghana (USD 4,100). This implies that prostaglandin analogue is not a cost-effective first-line medication for the treatment of POAG in Ghana.

\subsection{Sensitivity Analyses}

In the one-way sensitivity analysis, the ICER was not sensitive to the probability of developing asthma after the use of beta blockers (Appendix 4). Similarly, the ICER was neither sensitive to the age of the cohort nor the cost of prostaglandin analogues (Appendix 5 \& Appendix 6). In the two-way sensitivity analysis, however, the ICER was sensitive to the age of the cohort and the cost of prostaglandin analogues when varied simultaneously. When the age of the cohort was set at 41 years, and the cost of prostaglandin analogues at USD 13.00, prostaglandin analogues became cost-effective (Appendix 7). This implies that, generic

Table 5. Summary of Results.

\begin{tabular}{cccccc}
\hline Intervention & Cost (USD) & QALY & $\begin{array}{c}\text { Incremental Cost } \\
\text { (USD) }\end{array}$ & $\begin{array}{c}\text { Incremental } \\
\text { QALY }\end{array}$ & ICER \\
\hline $\begin{array}{c}\text { Beta Blockers } \\
\begin{array}{c}\text { Prostaglandin } \\
\text { analogues }\end{array}\end{array}$ & $2,663,700$ & 14,126 & - & - & - \\
\hline
\end{tabular}

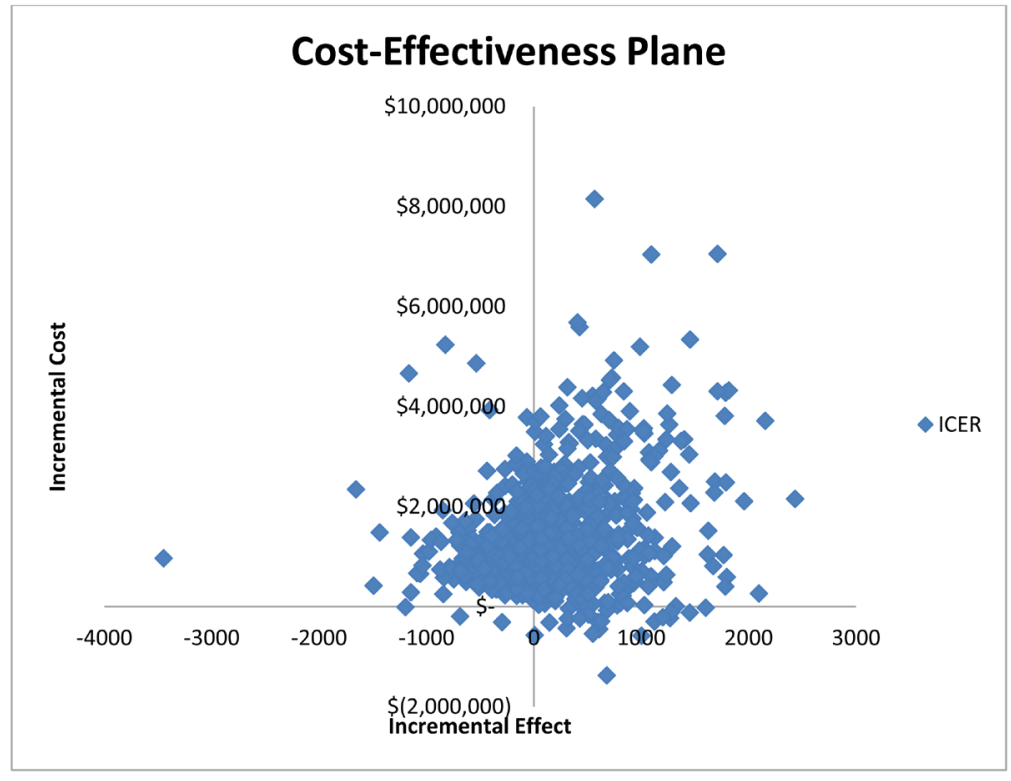

Figure 2. Cost-effectiveness plane. 
latanoprost (costing USD 13.00 per bottle) could be cost-effective for treating POAG patients 41 years old and below.

Results from the probabilistic sensitivity analysis were presented using a costeffectiveness plane, a cost-effectiveness acceptability curve (CEAC), and a costeffectiveness acceptability frontier (CEAF).

The cost-effectiveness plane (Figure 2) shows the spread of pairs of incremental costs $(\Delta \mathrm{C})$ and incremental effectiveness $(\Delta \mathrm{E})$ values from running 1,000 Monte Carlo simulations. The $\mathrm{x}$-axis shows incremental effectiveness, while the $y$-axis shows incremental cost. The slope of the line joining any point on the plane to the origin is equal to the incremental cost-effectiveness ratio (ICER). A cost-effectiveness plane has four quadrants: northeast (NE), southeast (SE), southwest (SW), and northwest (NW) (Briggs et al., 2006). A new intervention is said to dominate its comparator (more effective and less costly) if it is located in the SE quadrant. Conversely, a new intervention is said to be dominated by its comparator (less effective and more costly) if it is located on the NW quadrant. A new intervention is more effective and more costly if it is located in the NE quadrant, while a new intervention that is located in the SW quadrant is less effective and less costly.

In the cost-effectiveness plane, 977 (almost 98\%) of the cost-effectiveness pairs are concentrated on the northeast and northwest quadrants. This suggests that prostaglandin analogue is more costly than beta blockers, although this cannot be said with $100 \%$ certainty. The locations of the ICER points on the cost-effectiveness plane, however, makes it highly uncertain to tell whether prostaglandin analogue is more effective than beta blockers: $41 \%$ fell in the northwest and southwest quadrants, while $59 \%$ fell in the northeast and southeast quadrants.

In the cost-effectiveness acceptability curve (CEAC), presented in Figure 3, the $y$-axis indicates the probability that prostaglandin analogue is cost-effective compared to beta blockers, given a range of willingness-to-pay threshold values (on the $\mathrm{x}$-axis). At a willingness-to-pay threshold of USD 0 per QALY gained, the probability of prostaglandin analogues being cost-effective compared to beta blockers was 0.02 . The CEAC, however, shows that the probability of prostaglandin analogues being cost-effective increases as the willingness to pay for additional QALYs increases. When the willingness-to-pay threshold increases to USD 4,100, the probability of prostaglandin analogue being cost-effective compared to beta blockers increases to 0.27 . At the same willingness-to-pay threshold, the probability of beta blockers being cost-effective was 0.73 . This seems to suggest that prostaglandin analogue is not cost-effective compared to beta blockers. However, this cannot be said with absolute certainty since the probability of prostaglandin analogue being cost-effective is greater than 0 .

The cost-effectiveness acceptability frontier (CEAF) in Figure 4 indicates the probability that the alternative with the highest net-benefit will be cost-effective. Hence the decision uncertainty (or error probability) is 1 minus the value of the frontier. Like the CEAC, the CEAF shows that, at the willingness to pay threshold of USD 4100, the comparator (beta blockers) is cost-effective. The proba- 


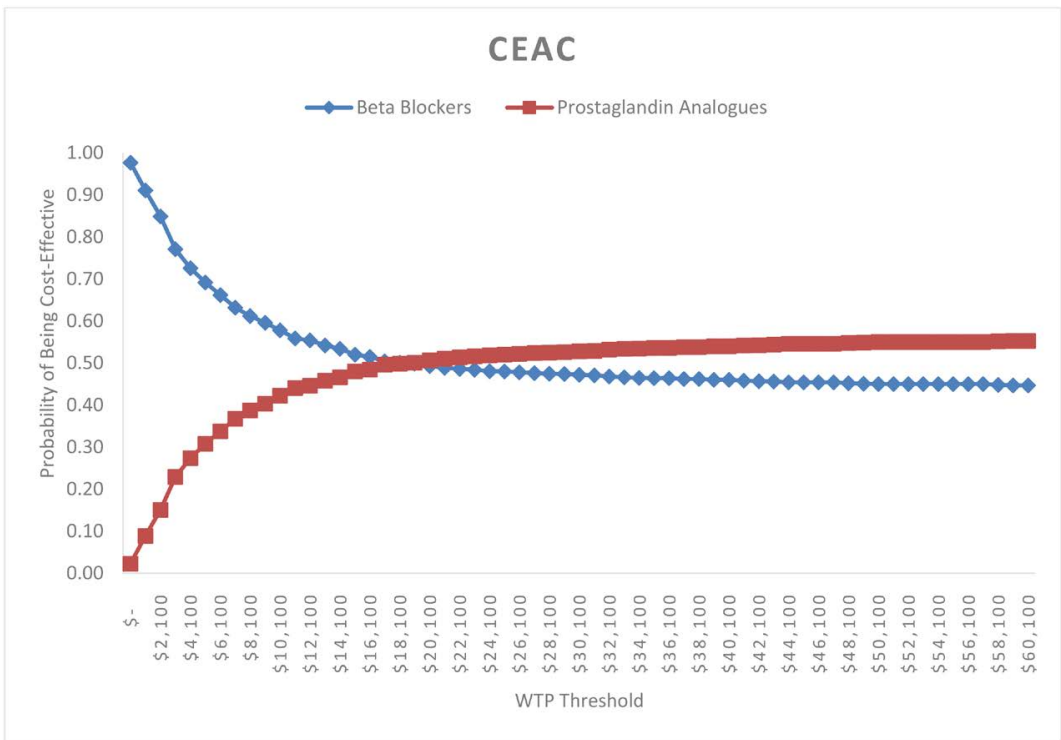

Figure 3. Cost-Effectiveness Acceptability Curve.

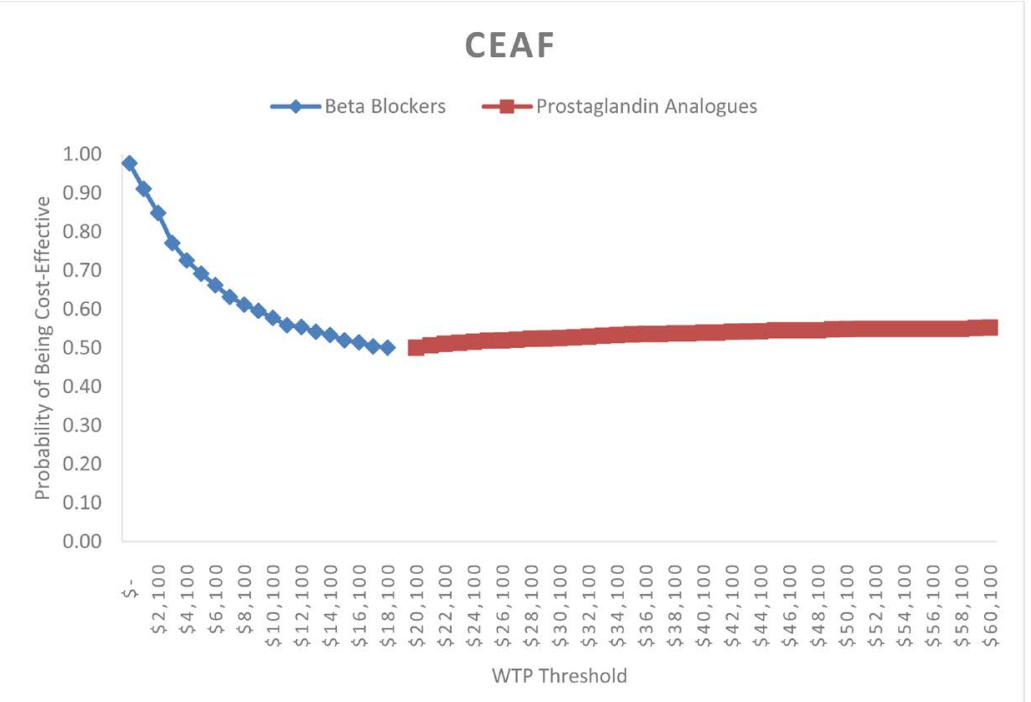

Figure 4. Cost-Effectiveness Acceptability Frontier.

bility of beta blockers being cost-effective is 0.73 with error probability of 0.27 .

\subsection{Expected Value of Perfect Information (EVPI)}

Figure 5 shows population EVPI for a range of willingness-to-pay threshold values between USD 0 and USD 60,100. Given a willingness to pay threshold of USD 4100, the population EVPI is USD 131 billion. This implies that further research would be potentially cost-effective if expected cost of further research is less than USD 131 billion. Beyond this value, further research is not worthwhile.

\section{Discussion}

There has been a growing concern in Ghana, recently, that anti-glaucoma medi- 


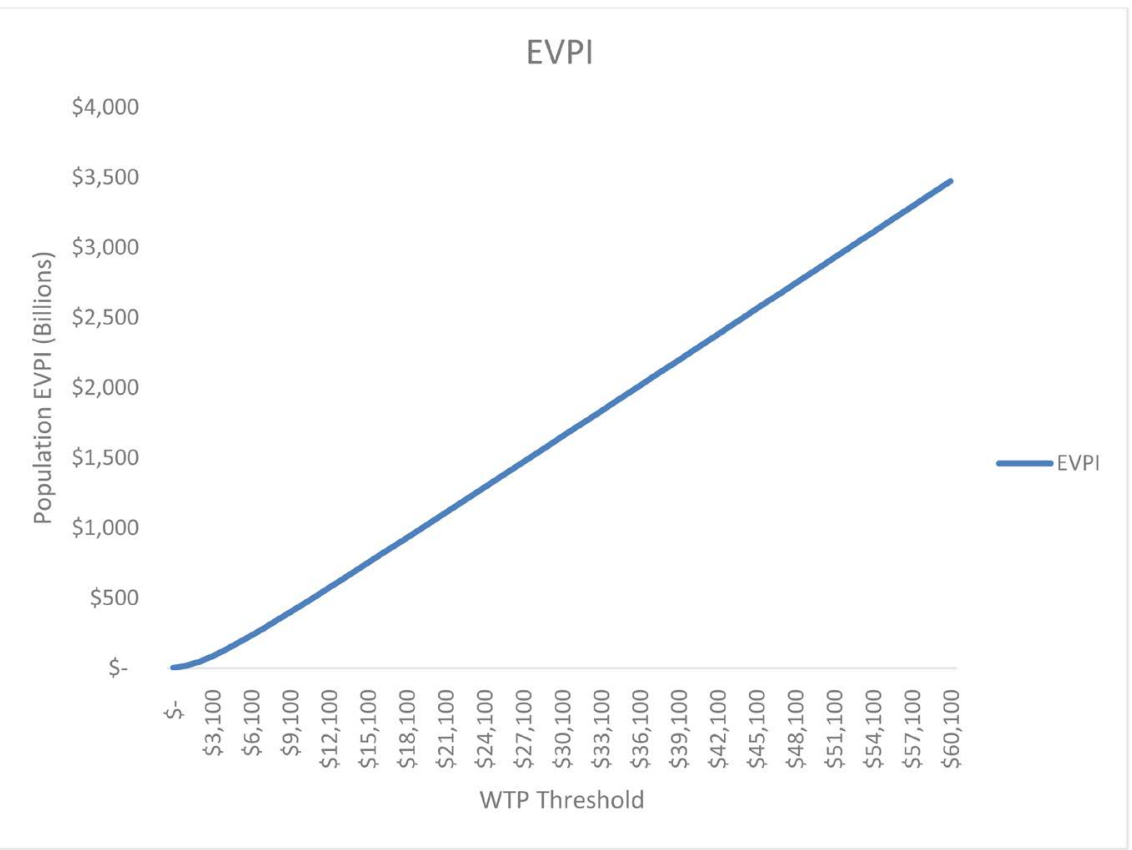

Figure 5. Population EVPI.

cations listed on the country's National Health Insurance Scheme's (NHIS) medicines list are not adequate to manage glaucoma. This has led to calls from many stakeholders for prostaglandin analogues, particularly latanoprost, to be added to the NHIS medicines list. However, the cost-effectiveness of prostaglandin analogues is yet to be established. The health care system of Ghana is faced with many challenges. Perhaps the greatest challenge that the health care system faces is inadequate funding. The resource constraints of the country make it highly important to establish the cost-effectiveness of prostaglandin analogues before recommending it to the National Health Insurance Authority. This study reports an economic evaluation modelling framework to compare the cost effectiveness of prostaglandin analogues with beta blockers as first line treatment for primary open angle glaucoma in Ghana.

\subsection{Cost-Utility Analysis}

Results from this study indicate that prostaglandin analogue is more effective, but also more costly, compared to beta blockers. The results also show that prostaglandin analogue is not a cost-effective alternative to beta blockers as a first-line medication for primary open angle glaucoma in Ghana, given the current economic situation of the country. A two-way sensitivity analysis on the age of the cohort and the cost of prostaglandin analogues (latanoprost), however, showed that generic latanoprost could be cost-effective for treating primary open angle glaucoma patients 41 years and below. This finding, especially with respect to age, seems to suggest that the full benefit of prostaglandin analogues can only be observed in the long run. Thus, even at USD 13.00 per bottle, prostaglandin analogue was not cost-effective for the cohort used in the base-case 
analysis (55 years) because the cohort had a higher probability of death, and therefore died out too quickly to benefit fully from the intervention. However, it became cost-effective for a younger cohort (41 years and below), with a lower probability of death.

The probabilistic sensitivity analysis reveals some level of uncertainty surrounding the results of the cost-effectiveness analysis. Results from EVPI suggest that further evidence would be necessary to reduce decision uncertainty provided that the expected cost of further research does not exceed USD 131 billion.

\subsection{Related Studies}

There are several studies that seek to establish the cost-effectiveness of treatment interventions for glaucoma. However, studies that compare different anti-glaucoma medications are limited. In Ghana, economic evaluation studies on glaucoma treatment are very scanty. Kuffuor et al. [7], who established the efficacy of anti-glaucoma medications in Ghana, did not account for their costs. Wittenborn \& Rein [19] also compared the cost-effectiveness of glaucoma interventions in Barbados and Ghana, but did not consider the cost-effectiveness between different ant-glaucoma medications. Thus, studies that establish the cost-effectiveness of anti-glaucoma medications in Ghana are almost non-existent. As far as I know, this is the first study that seeks to establish the cost-effectiveness between anti-glaucoma medications for treating primary open angle glaucoma in Ghana. The model reported in this study, therefore, provides a relevant framework for further studies on glaucoma treatment costs and health gains.

\subsection{Limitations of the Study}

The study has several limitations, which could have influenced the incremental cost and/or incremental QALY, and for that matter the ICER. The limitations stem mainly from the key assumptions of the model.

As far as medical treatment is concerned, the only adverse event I considered was asthma. All other adverse events were assumed to be negligible since they could be promptly treated. This assumption may have led to underestimation of costs and overestimation of QALYs under both interventions. It is, however, difficult to tell which direction this limitation would have driven the ICER. The direction of the effect would depend on the specific adverse events associated with each intervention, the reduction in QALYs due to each adverse event, and the cost involved in treating the respective events.

Besides, all post-surgery complications were assumed to be negligible. Clearly, this assumption might have led to underestimation of costs in both groups. However, the possible impact of this limitation on the ICER would depend on how many patients in each intervention group had complications after undergoing surgery.

It was further assumed that POAG patients would not need any anti-glaucoma medication after they have undergone surgery. In practice, however, some 
POAG patients may have elevated IOP even after surgery, and would therefore need further medical treatment. Ignoring this probability might have led to understatement of costs in both groups.

Furthermore, I assumed no extra follow-up costs for POAG patients who developed asthma after the use of beta blockers. This assumption might have led to underestimation of costs associated with beta blockers and, hence, overestimation of the ICER.

I also assumed that no patient died from asthma that resulted from the use of beta blockers. Neglecting possible asthma-related deaths could have led to overestimation of QALYs gained under beta blockers and, therefore, underestimation of incremental QALYs, and eventually overestimation of the ICER.

Another limitation of the study stems from the assumption that there was no shift in treatment before a patient progressed from early POAG stage. In practice, patients at early POAG stage who show intolerance to beta blockers or prostaglandin analogues may be shifted to another treatment before they progress to the next stage. This assumption might, therefore, have led to underestimation of costs under both interventions.

Utility measures used in the model may not be accurate. The health utilities associated with the POAG stages were retrieved from the National Collaborating Centre for Acute Care (UK) [16]. According to the authors, however, the methodology adopted to estimate the values had not yet been validated. Besides, the original health utilities were estimated for different ocular conditions causing a defect in visual acuity, and might not be applicable to glaucoma patients since the pattern of visual loss in glaucoma differs from other conditions.

Probability of death could have also been underestimated under both interventions. In the absence of data to show that people die from glaucoma, I assumed that the probability of death among POAG patients was the same as that for the general population of Ghana. This assumption ignores the fact that the blind and people with visual impairment may have a higher probability of death from accidents.

I assumed that all patients who progressed from early POAG stage were given combination therapy. However, experts stated that some patients are shifted from one monotherapy to another before they are eventually given combination therapy, although the percentage of patients who fall into this category could not be estimated.

In addition, the assumption that the severity of the POAG condition is similar in both eyes of a patient may not reflect reality since, in practice, a patient could present with unilateral POAG.

Finally, the WTP threshold adopted in the study may not reflect the actual WTP threshold in Ghana. While the WTP threshold used in the model was based on DALYs averted, the study measured health effect in terms of QALYs gained. QALYs gained and DALYs averted may not be interchangeable since they are partly based on different assumptions and different methodologies. 


\subsection{Conclusion}

Given the existing evidence, prostaglandin analogue is not a cost-effective alternative to beta blockers as a first-line medication for primary open angle glaucoma in Ghana. Generic latanoprost may, however, be cost-effective in treating POAG patients 41 years and younger. The population EVPI shows that further research to reduce decision uncertainty would be cost-effective if expected cost of further research does not exceed USD 131 billion.

\section{References}

[1] Potter, A., Debrah, O., Ashun, J. and Blanchet, K. (2013) Eye Health Systems Assessment (EHSA): Ghana Country Report.

[2] World Glaucoma Association (2010) WGA African Glaucoma Summit: The 1st African Glaucoma Summit.

[3] Quigley, H.A. and Broman, A.T. (2006) The Number of People with Glaucoma Worldwide in 2010 and 2020. British Journal of Ophthalmology, 90, 262-267. https://doi.org/10.1136/bjo.2005.081224

[4] Guzek, J.P., Anyomi, F.K., Fiadoyor, S. and Nyonator, F. (2005) Prevalence of Blindness in People over 40 Years in the Volta Region of Ghana. Ghana Medical Journal, 39, 55-62.

[5] About 700,000 People in Ghana Suffer Glaucoma. http://www.ghanaweb.com/GhanaHomePage/health/About-700-000-people-in-Gha na-suffer-glaucoma-349940

[6] Cook, C. (2009) Glaucoma in Africa: Size of the Problem and Possible Solutions. Journal of Glaucoma, 18, 124-128. https://doi.org/10.1097/IJG.0b013e318189158c

[7] Koffuor, G.A., Ababio-Danso, B., Gyanfosu, L. and Amoateng, P. (2012) The Efficacy of NHIS-Listed Anti-Glaucoma Drugs in the Management of Primary Open-Angle Glaucoma. Journal of Medical and Biomedical Sciences, 1.

[8] Ministry of Health (Ghana) (2010) Standard Treatment Guidelines. 6th Edition, Yamens Press Ltd., Accra.

[9] Rathore, K.S. and Nema, R.K. (2009) Medical Management of Glaucoma: An Overview. International Journal of Pharm Tech Research, 1, 863-869.

[10] Thylefors, B. and Négrel, A.D. (1994) The Global Impact of Glaucoma. Bulletin of the World Health Organization, 72, 323-326.

[11] Ophthalmology, A.A.O. (2009) Practical Ophthalmology: A Manual for Beginning Residents. 6th Edition, 6th Revised Edition, American Academy of Ophthalmology, San Francisco.

[12] Carroll, N.J. and Johnson, C.A. (2013) Visual Field Testing: From One Medical Student to Another. http://webeye.ophth.uiowa.edu/eyeforum/tutorials/VF-testing/

[13] Shafranov, G. (2011) Essentials of Automated Perimetry. In: Walsh, T.J., Ed., Visual Fields Examination and Interpretation, 3rd Edition, Oxford University Press, Oxford.

[14] Heijl, A., et al. (2002) Reduction of Intraocular Pressure and Glaucoma Progression: Results from the Early Manifest Glaucoma Trial. Archives of Ophthalmology, 120, 1268-1279.

[15] Ting, N.S., Li Yim, J.F. and Ng, J.Y. (2014) Different Strategies and Cost-Effectiveness in the Treatment of Primary Open Angle Glaucoma. ClinicoEconomics and Outcomes Research, 6, 523-530. 
[16] National Collaborating Centre for Acute Care (UK) (2009) Glaucoma: Diagnosis and Management of Chronic Open Angle Glaucoma and Ocular Hypertension. National Collaborating Centre for Acute Care (UK), London.

[17] Government of Ghana (2003) National Health Insurance Act, 2003. Ghana Publishing Company, Accra.

[18] National Health Insurance Authority (Ghana) (2011) 2011 Annual Report.

[19] Wittenborn, J.S. and Rein, D.B. (2011) Cost-Effectiveness of Glaucoma Interventions in Barbados and Ghana. Optometry and Vision Science Journal, 88, 155-163. https://doi.org/10.1097/OPX.0b013e3181fc30f3

[20] Ghana Home. http://www.worldbank.org/en/country/ghana

[21] GHO. http://apps.who.int/gho/data/node.main.688?lang=en

[22] World Health Organization (2015) Ghana: WHO Statistical Profile. http://www.who.int/gho/countries/gha.pdf?ua=1

[23] Ghana Statistical Service (2014) Ghana Living Standards Survey round 6 (GLSS6): Poverty Profile in Ghana (2005-2013).

[24] WHO. http://www.who.int/countries/gha/en/

[25] Burr, J.M., et al. (2007) The Clinical Effectiveness and Cost-Effectiveness of Screening for Open Angle Glaucoma: A Systematic Review and Economic Evaluation. Health Technology Assessment, 11, 1-190.

[26] Leske, M.C., et al. (2007) Predictors of Long-Term Progression in the Early Manifest Glaucoma Trial. Ophthalmology, 114, 1965-1972.

[27] Kirwan, J.F., Nightingale, J.A., Bunce, C. and Wormald, R. (2002) Beta Blockers for Glaucoma and Excess Risk of Airways Obstruction: Population Based Cohort Study. BMJ, 325, 1396-1397. https://doi.org/10.1136/bmj.325.7377.1396

[28] GHO. http://apps.who.int/gho/data/view.main.60630

[29] Van Gestel, A., et al. (2010) The Relationship between Visual Field Loss in Glaucoma and Health-Related Quality-of-Life. Eye, 24, 1759-1769. https://doi.org/10.1038/eye.2010.133

[30] Béchetoille, A., et al. (2008) Measurement of Health-Related Quality of Life with Glaucoma: Validation of the Glau-QoL 36-Item Questionnaire. Acta Ophthalmologica, 86, 71-80. https://doi.org/10.1111/j.1600-0420.2007.00999.x

[31] Rein, D.B., Wirth, K.E., Johnson, C.A. and Lee, P.P. (2007) Estimating Quality-Adjusted Life Year Losses Associated with Visual Field Deficits using Methodological Approaches. Ophthalmic Epidemiology, 14, 258-264. https://doi.org/10.1080/01658100701473267

[32] Brown, M.M., Brown, G.C., Sharma, S. and Landy, J. (2003) Health Care Economic Analyses and Value-Based Medicine. Survey of Ophthalmology, 48, 204-223.

[33] Schermer, T.R., et al. (2002) Randomized Controlled Economic Evaluation of Asthma Self-Management in Primary Health Care. American Journal of Respiratory and Critical Care Medicine, 166, 1062-1072. https://doi.org/10.1164/rccm.2105116

[34] Chen, H., et al. (2007) Asthma Control, Severity, and Quality of Life: Quantifying the Effect of Uncontrolled Disease. Journal of Allergy and Clinical Immunology, 120, 396-402.

[35] Drummond, M.F., Torrance, G.W., O’Brien, B.J. and Stoddart, G.L. (1997) Methods for the Economic Evaluation of Health Care Programmes. 2nd Edition, Oxford University Press, Oxford.

[36] Gold, M.R., Siegel, J.E., Russel, L.B. and Weinstein, M.C. (1996) Cost-Effectiveness 
in Health and Medicine. Oxford University Press, New York.

[37] Johns, B., Baltussen, R. and Hutubessy, R. (2003) Programme Costs in the Economic Evaluation of Health Interventions. Cost Effectiveness and Resource Allocation, 1, 1. https://doi.org/10.1186/1478-7547-1-1

[38] Medicines List. http://www.nhis.gov.gh/medlist.aspx

[39] National Health Insurance Scheme (Ghana) (2016a) G-DRG Revised Tariffs v 2016: Tariffs for CHAG Primary Care Hospital (Catering Exclusive).

[40] National Health Insurance Scheme (Ghana) (2016b) G-DRG Revised Tariffs v 2016: Tariffs for Public Primary Care Hospital (catering exclusive).

[41] World Health Organization (2003) Making Choices in Health: WHO Guide to Cost-Effectiveness Analysis. World Health Organization, Geneva.

[42] Briggs, A.H., Claxton, K. and Sculpher, M.J. (2006) Decision Modelling for Health Economic Evaluation. Oxford University Press, Oxford. 
Appendix 1. Mean Reduction in IOP from Baseline per Intervention.

\begin{tabular}{cc}
\hline Intervention & Mean Reduction \\
\hline Beta Blockers & $2.88 \mathrm{mmHg}^{*}$ \\
Prostaglandin Analogues & $4.2 \mathrm{mmHg}$ \\
\hline
\end{tabular}

${ }^{*} \mathrm{mmHg}$ (millimetres of mercury) is the unit of measure for intraocular pressure Source: (National Collaborating Centre for Acute Care (UK), 2009).

Appendix 2. Life Table for Ghana 2013.

\begin{tabular}{|c|c|c|}
\hline \multirow{2}{*}{ Age } & \multicolumn{2}{|c|}{ Probability of Death } \\
\hline & Female & Male \\
\hline$<1$ year & 0.042299 & 0.0521 \\
\hline $1-4$ years & 0.022923 & 0.024086 \\
\hline $5-9$ years & 0.016682 & 0.016784 \\
\hline $10-14$ years & 0.010198 & 0.009866 \\
\hline 15 - 19 years & 0.011471 & 0.013503 \\
\hline $20-24$ years & 0.014199 & 0.01904 \\
\hline $25-29$ years & 0.016908 & 0.020019 \\
\hline $30-34$ years & 0.01927 & 0.021748 \\
\hline 35 - 39 years & 0.023739 & 0.026648 \\
\hline $40-44$ years & 0.027454 & 0.030997 \\
\hline $45-49$ years & 0.032665 & 0.039465 \\
\hline $50-54$ years & 0.04376 & 0.054715 \\
\hline $55-59$ years & 0.056971 & 0.069616 \\
\hline $60-64$ years & 0.084521 & 0.101838 \\
\hline $65-69$ years & 0.131772 & 0.151119 \\
\hline $70-74$ years & 0.208545 & 0.228736 \\
\hline $75-79$ years & 0.322648 & 0.345576 \\
\hline $80-84$ years & 0.489874 & 0.520373 \\
\hline $85-89$ years & 0.701343 & 0.715682 \\
\hline 90 - 94 years & 0.92976 & 0.947176 \\
\hline 95 - 99 years & 0.969188 & 0.975211 \\
\hline $100+$ years & 1 & 1 \\
\hline $100+$ years & 1 & 1 \\
\hline
\end{tabular}

Source: http://apps.who.int/gho/data/view.main.60630.

Appendix 3. Unit costs of resources.

\begin{tabular}{cccc}
\hline Resource & Unit of Pricing & Price (USD) & Source \\
\hline Acetazolamide Tablet, 250 mg & Tablet & 0.06 & {$[38]$} \\
Pilocarpine Eye Drops & Bottle & $3.38(\mathrm{a})$ & {$[38]$} \\
Timolol Maleate Eye Drops, 0.5\% & Bottle & 1.88 & {$[38]$} \\
Latanoprost & Bottle & $25.77(\mathrm{~b})$ & Expert opinion \\
Trabeculectomy & 1 Procedure & 34.53 & {$[39][40]$} \\
Budesonide DPI, 200 micrograms & Inhaler(d) & 18.82 & {$[38]$} \\
Salbutamol Inhaler, 100 micrograms & Inhaler(d) & 4.59 & {$[38]$} \\
Fluticasone MDI & Inhaler(d) & $18.72(\mathrm{c})$ & {$[38]$} \\
Beclometasone dipropionate Inhaler, 100 micrograms & Inhaler(d) & 16.31 & {$[38]$} \\
\hline
\end{tabular}

(a) Average cost for Pilocarpine Eye Drops $2 \%$ and Pilocarpine Eye Drops $4 \%$ (see appendix 2) (b) Average cost of generic latanoprost and branded latanoprost (Xalatan) (c) Average cost for Fluticasone MDI 125 microgram and Fluticasone MDI 250 microgram (see appendix 2) (d) All inhalers are for the treatment of asthma. 
Appendix 4. One-way Sensitivity Analysis on Probability of Developing Asthma.

\begin{tabular}{cccc}
\hline Annual Probalility of asthma & Incr. Cost & Incr. QALY & ICER \\
\hline $\mathbf{0 . 0 0}$ & 1223426 & 104 & 11746 \\
$\mathbf{0 . 0 1}$ & 1223151 & 104 & 11715 \\
$\mathbf{0 . 0 2}$ & 1222873 & 105 & 11684 \\
$\mathbf{0 . 0 3}$ & 1222593 & 105 & 11653 \\
$\mathbf{0 . 0 4}$ & 1222310 & 105 & 11622 \\
$\mathbf{0 . 0 5}$ & 1222025 & 105 & 11591 \\
$\mathbf{0 . 0 6}$ & 1221737 & 106 & 11559 \\
\hline
\end{tabular}

Appendix 5. One-way Sensitivity Analysis on Cost of Latanoprost (Excerpt).

\begin{tabular}{cccc}
\hline Cost of Prostaglandin Analogues & Incr. Cost & Incr. QALY & ICER \\
\hline 13 & 506494 & 105 & 4821 \\
14 & 562559 & 105 & 5355 \\
15 & 618624 & 105 & 5888 \\
16 & 674688 & 105 & 6422 \\
17 & 730753 & 105 & 6956 \\
18 & 786817 & 105 & 7489 \\
19 & 842882 & 105 & 8023 \\
20 & 898946 & 105 & 8557 \\
21 & 955011 & 105 & 9090 \\
22 & 1011075 & 105 & 9624 \\
23 & 1067140 & 105 & 10158 \\
25 & 1123204 & 105 & 10691 \\
\end{tabular}


Appendix 6. One-way Sensitivity Analysis on Age of the Cohort (Ercerpt).

\begin{tabular}{|c|c|c|c|}
\hline Age in Years & Incr. Cost & Incr. QALY & ICER \\
\hline 30 & 1295960 & 136 & 9495 \\
\hline 31 & 1295090 & 136 & 9517 \\
\hline 32 & 1294006 & 136 & 9541 \\
\hline 33 & 1292658 & 135 & 9567 \\
\hline 34 & 1290985 & 135 & 9594 \\
\hline 35 & 1289583 & 134 & 9622 \\
\hline 36 & 1288580 & 133 & 9654 \\
\hline 37 & 1287326 & 133 & 9690 \\
\hline 38 & 1285765 & 132 & 9730 \\
\hline 39 & 1283826 & 131 & 9773 \\
\hline 40 & 1281997 & 131 & 9819 \\
\hline 41 & 1280350 & 130 & 9872 \\
\hline 42 & 1278289 & 129 & 9931 \\
\hline 43 & 1275720 & 128 & 9997 \\
\hline 44 & 1272526 & 126 & 10068 \\
\hline 45 & 1269536 & 125 & 10145 \\
\hline 46 & 1266879 & 124 & 10233 \\
\hline 47 & 1263554 & 122 & 10332 \\
\hline 48 & 1259409 & 121 & 10442 \\
\hline 49 & 1254252 & 119 & 10561 \\
\hline 50 & 1249717 & 117 & 10690 \\
\hline 51 & 1246130 & 115 & 10839 \\
\hline 52 & 1241643 & 113 & 11010 \\
\hline 53 & 1236054 & 110 & 11200 \\
\hline 54 & 1229102 & 108 & 11408 \\
\hline 55 & 1222439 & 105 & 11636 \\
\hline 56 & 1216307 & 102 & 11899 \\
\hline 57 & 1208639 & 99 & 12196 \\
\hline 58 & 1199070 & 96 & 12527 \\
\hline 59 & 1187133 & 92 & 12887 \\
\hline 60 & 1176394 & 89 & 13277 \\
\hline 61 & 1167576 & 85 & 13726 \\
\hline 62 & 1156515 & 81 & 14231 \\
\hline 63 & 1142638 & 77 & 14790 \\
\hline 64 & 1125211 & 73 & 15396 \\
\hline 65 & 1109913 & 69 & 16051 \\
\hline 66 & 1098006 & 65 & 16797 \\
\hline
\end{tabular}


Appendix 7. Two-way Sensitivity Analysis on Age of Cohort and Cost of Latanoprost (Excerpt).

\begin{tabular}{|c|c|c|c|c|c|c|}
\hline \multirow{2}{*}{ Age in Years } & \multicolumn{6}{|c|}{ Cost of Latanoprost (USD) } \\
\hline & 13 & 14 & 15 & 16 & 17 & 18 \\
\hline 30 & 3922 & 4359 & 4795 & 5231 & 5668 & 6104 \\
\hline 31 & 3931 & 4369 & 4806 & 5244 & 5681 & 6118 \\
\hline 32 & 3942 & 4380 & 4819 & 5257 & 5696 & 6134 \\
\hline 33 & 3952 & 4392 & 4832 & 5271 & 5711 & 6151 \\
\hline 34 & 3964 & 4405 & 4846 & 5286 & 5727 & 6168 \\
\hline 35 & 3976 & 4418 & 4860 & 5302 & 5744 & 6186 \\
\hline 36 & 3989 & 4433 & 4876 & 5320 & 5764 & 6207 \\
\hline 37 & 4004 & 4449 & 4895 & 5340 & 5785 & 6231 \\
\hline 38 & 4021 & 4468 & 4915 & 5362 & 5809 & 6256 \\
\hline 39 & 4039 & 4488 & 4937 & 5386 & 5835 & 6284 \\
\hline 40 & 4058 & 4509 & 4960 & 5411 & 5863 & 6314 \\
\hline 41 & 4080 & 4534 & 4987 & 5441 & 5894 & 6348 \\
\hline 42 & 4105 & 4561 & 5018 & 5474 & 5930 & 6386 \\
\hline 43 & 4133 & 4592 & 5051 & 5511 & 5970 & 6429 \\
\hline 44 & 4163 & 4625 & 5088 & 5550 & 6012 & 6475 \\
\hline 45 & 4195 & 4661 & 5126 & 5592 & 6058 & 6524 \\
\hline 46 & 4232 & 4701 & 5171 & 5641 & 6111 & 6581 \\
\hline 47 & 4273 & 4748 & 5222 & 5697 & 6171 & 6646 \\
\hline 48 & 4319 & 4799 & 5278 & 5758 & 6237 & 6717 \\
\hline 49 & 4369 & 4854 & 5339 & 5824 & 6309 & 6794 \\
\hline 50 & 4423 & 4914 & 5405 & 5895 & 6386 & 6877 \\
\hline
\end{tabular}

